\title{
A Local Model of Eye Adaptation for High Dynamic Range Images
}

\author{
Patrick Ledda \\ University of Bristol, UK \\ ledda@cs.bris.ac.uk
}

\author{
Luis Paulo Santos \\ University of Minho, Portugal \\ psantos@di.uminho.pt
}

\author{
Alan Chalmers \\ University of Bristol, UK \\ alan@cs.bris.ac.uk
}

\begin{abstract}
In the real world, the human eye is confronted with a wide range of luminances from bright sunshine to low night light. Our eyes cope with this vast range of intensities by adaptation; changing their sensitivity to be responsive at different illumination levels. This adaptation is highly localized, allowing us to see both dark and bright regions of a high dynamic range environment. In this paper we present a new model of eye adaptation based on physiological data. The model, which can be easily integrated into existing renderers, can function either as a static local tone mapping operator for single high dynamic range image, or as a temporal adaptation model taking into account time elapsed and intensity of preadaptation for a dynamic sequence. We finally validate our technique with a high dynamic range display and a psychophysical study.
\end{abstract}

\section{Categories and Subject Descriptors}

I.3.3 [Computer Graphics]: Picture/Image GenerationDisplay Algorithms

\section{Keywords}

Human Visual System, Eye Adaptation, High Dynamic Range, Tone Mapping

\section{INTRODUCTION}

High fidelity renderings of real scenes should accurately reproduce any large dynamic range of luminances that may be present in reality. This is particularly important for applications in which an accurate representation of the illumination is critical for the correct perception of the environment, for example visibility in driving simulators or the perception of archaeological sites (Figure 1).

Although humans are capable of seeing a huge range of intensities, from daylight levels of around $10^{8} \mathrm{~cd} / \mathrm{m}^{2}$ to night luminances of approximately $10^{-6} \mathrm{~cd} / \mathrm{m}^{2}$, the cells of the Human Visual System (HVS) have a much more limited

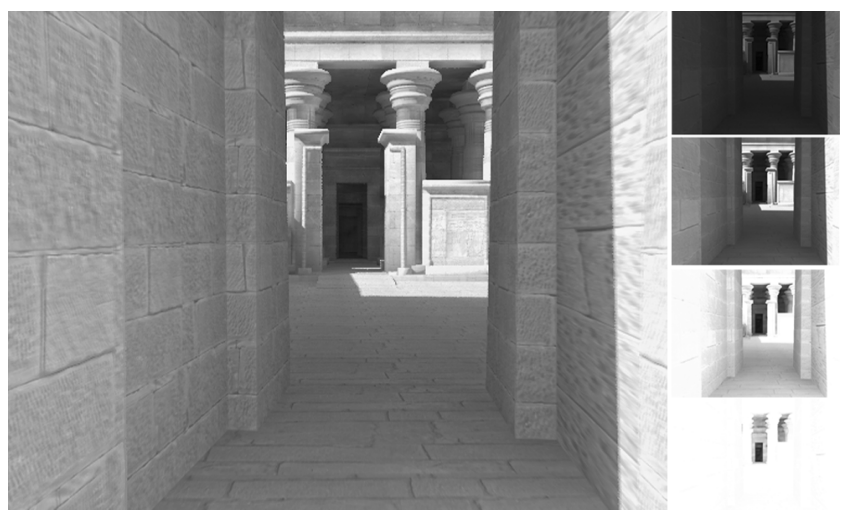

Figure 1: On the left, the Kalabsha temple tonemapped with our local model. On the right, linear mappings at different exposure times.

response range. Our visual system copes with the large luminance range by a process called adaptation. At the retina level, eye adaptation is highly localized allowing us to see both dark and bright regions in the same high dynamic range environment. Adaptation is not instantaneous and in the case of, for instance, switching the lights off in a room, this process may take many minutes.

In this paper we present a new local model of adaptation based on the response of the photoreceptors in the retina. After computing local adaptation level across an image, we generate separate response signals for rods and cones at a pixel level. We then compute the time-course of light and dark adaptation by applying a model derived from psychophysical data. This allows us to not only tone map individual images based on perceptual data, but, as the model is time dependent, also to simulate adaptation over time of a scene.

Although it is possible to generate high dynamic range images, for example using methods proposed in [5] or render them using systems such as Radiance [35], most modern display devices are still only capable of displaying images with contrast ratios of less than two orders of magnitude. Tone mapping operators are thus typically used to generate a low contrast image which should be perceived in a similar way as if the viewer were standing in the real scene. Recently, a number of novel high dynamic range devices are beginning 
to appear capable of huge contrast ratios. We validate our adaptation model against reality using such a high dynamic range display device.

\section{PREVIOUS WORK}

According to a framework proposed by Tumblin and Rushmeier [31], tone mapping operators (TMO) aim to generate images visually similar to a real scene by careful mapping to a set of luminances that can be displayed on a low contrast ratio display or printed. These operators can be divided into different categories depending on how they attempt to reduce contrast of an HDR map. Those models such as $[34,27,17,30,7]$ that apply the same mapping function across the image are known as global operators. These algorithms, although not very expensive, do not cope sufficiently well with images that exhibit detail across a large range of luminances. Those operators in which the mapping varies spatially depending on a neighborhood of a pixel are known as local. Local operators are generally capable of a greater contrast reduction allowing significant compression of the dynamic range of a scene whilst preserving detail. A major issue with local operators is that contrast reversals (or "halos") artifacts appear around high contrast edges. See for instance $[3,27,23,16,30]$. To overcome this problem several approaches have been proposed $[21,1,25,10,22]$ that employ a series of band-pass filters or apply a multiscale decomposition into intrinsic layers, such as reflectance, illumination, texture, etc. Contrast reduction is then applied to the illumination layer. Other authors focus on robust statistical estimators to improve current local techniques $[32,6$, $9,4]$. In addition, some operators try to mimic the human visual system using mapping functions that closely resemble aspects of human vision. [33, 31, 34, 17, 23, 32].

Most tone mapping operators have been developed for static images, whereas a few global operators, including [26, 24, 8] are able to support animated sequences. Finally, few perceptual operators [11, 24, 8], also model other effects such as the time course of adaptation, loss of color and visual acuity at different illumination levels aiming to produce images which are even closer to what an observer would perceive in reality. Unfortunately, these global models are not always capable of sufficiently compressing the dynamic range of a high contrast scene whilst preserving detail.

In this paper, we present a new local and time dependent model of visual adaptation. The operator is based on computing retinal response of rods and cones in a similar way proposed by Pattanaik et al. [24]. In their model a global response is computed from a unique adaptation level which, although very fast, is not capable of huge contrast reduction. We base our model on their work, however, we make some important extensions:

1. Adaptation is highly localized therefore a local model of adaptation is introduced. This is then used to compute a local response

2. The local model allows us to effectively adapt each pixel differently based on a neighborhood of values. This gives us the advantage of being able to simulate, for example, the stimulation of only cones in one region and only rods in another.
3. The local model has obvious advantages when tone mapping the input image as it does not suffer loss of contrast for images that exhibit detail across a wide range of luminance levels.

4. Our model simulates the time-course of adaptation as a function of preadaptation time and intensity as well as postadaptation intensity.

5. We are also able to simulate change of color sensitivity as a function of luminance level.

\section{SENSITIVITY OF THE EYE}

There are two types of photoreceptors in our retina which allow humans to make visual discrimination over an extremely wide range of light intensities. The rods are highly sensitive photoreceptors and are used for vision under dark and dim conditions. They do not mediate color vision and have a poor visual acuity. The cones on the other hand are active at much higher light levels and are responsible for color vision. The cones also provide us with our highest visual acuity. The enormous range the visual system can deal with may be subdivided in two main regions of similar size: photopic (cone-mediated and range between $10^{1} \mathrm{~cd} / \mathrm{m}^{2}$ to $10^{8} \mathrm{~cd} / \mathrm{m}^{2}$ ) and scotopic (rod-mediated, between $10^{-1}$ to $10^{-6} \mathrm{~cd} / \mathrm{m}^{2}$ ). Those light levels that stimulate both types of receptors belong to the (not well studied) mesopic range.

\subsection{Psychophysical Measurements}

The eye operates over a large range of light levels, however the way in which we process visual stimuli differs. Under low light levels, for example, the sensitivity of the eye is greatly increased and light that would supply as little as 100 quanta (light energy) may be discriminated against a black background [14]. In the photopic range however, our eye would require thousands or millions of quanta for the light to be seen against a background of higher illumination. The sensitivity of our eye can be measured by determining the absolute intensity threshold, that is, the minimum luminance of a test spot required to produce a visual sensation. This can be achieved by placing a subject in front of a background wall of a given intensity, and increasing the luminance of the test spot until the subject reports its presence. This type of psychophysical experiment is known as threshold versus intensity (tvi) experiment. The tvi of the human visual system is shown in Figure 2. As can be seen, as the prevailing luminance (Adapting Intensity) increases, the visual system becomes less sensitive to light (Threshold Intensity increases). The intensity required for the detection of the test spot increases with the increase of background (or ambient) illumination. Depending on the background luminance level, test size and retinal eccentricity a one or two branched curve is obtained. When the intensity discrimination of the rod system is isolated, for example, three main sections of the curves become apparent. At the lowest intensity levels the threshold is unaffected by the background light and as the luminance is increased, the slope of the curve remains constant. When the background level is increased above 2 $\log$ scotopic trolands (troland = luminance/pupilarea), the threshold rises rapidly and the rod system starts to become incapable of detecting any stimulus. This is known as saturation and is shown in Figure 2 as a dotted line. For a wide range of background levels the slope of the curve is close to 


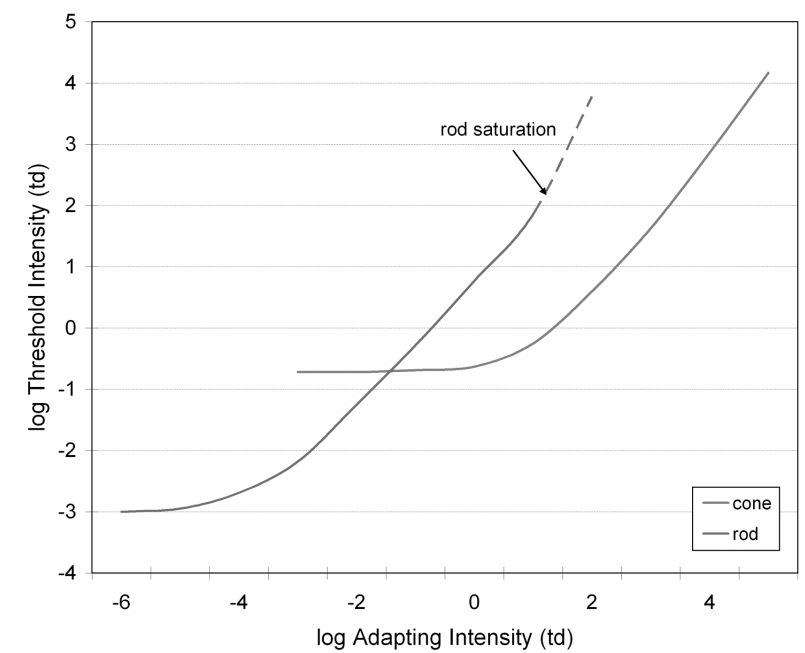

Figure 2: Threshold vs Intensity curve, after Hood [14]. As the background intensity increases, the detection threshold becomes higher.

1.0 and the contrast threshold is constant as the intensity level increases.

\subsection{Response and Adaptation Mechanisms}

There are various mechanisms in the eye that can cause the change in sensitivity as well as other mechanisms that help to maintain constant sensitivity. These mechanisms are responsible for keeping the system responsive at different light intensities. By looking at retinal response we can understand this concept a little better. At low adaptation levels for example, the cellular response to even low light levels, rapidly increases. For more intense flashes of light, however, the response increment starts decreasing and eventually it reaches a point where no intensity will produce a response. This is known as response compression. According to this model, response compression would have a dramatic effect on our visual system upon adapting fields of higher intensities causing a desensitization of our visual system. At higher adaptation levels the response would be extremely reduced and we would be blind even at moderate adapting intensities. This is obviously not true thanks to a series of mechanisms that maintain the HVS responsiveness by acting to scale the incoming light by some multiplicative (mainly) and subtractive constant. Factors such as pupil change, pigment depletion, subtractive and other multiplicative mechanisms are all responsible for maintaining the sensitivity of the system. More details of the physiology of the eye can be found in [14].

\section{OUR MODEL}

Similarly to Pattanaik et al. [24], our model is based on an electrophysiological model that predicts at any given adaptation level the response of the rods and cones. This model was first proposed by Naka and Rushton [20] and subsequently used by other authors to psychophysically model brightness perception [36]. The response $R$ has the form:

$$
R(I)=I^{n} /\left(I^{n}+\sigma^{n}\right)
$$

where $I$ is the test flash intensity and $\sigma$ is the half-saturation parameter (i.e. the value that causes half of the system's response, $R=0.5$ ) and $n$ is a sensitivity constant. Different values for $n$ have been proposed by authors, normally $(0.7<n<2.0)$ [36]. This response model incorporates the multiplicative mechanism described in the previous section by a change in $\sigma$ with varying illumination levels. Figure 3 shows how the response is affected by different adaptation levels due to multiplicative mechanisms which help the HVS to be responsive even at the highest luminances.

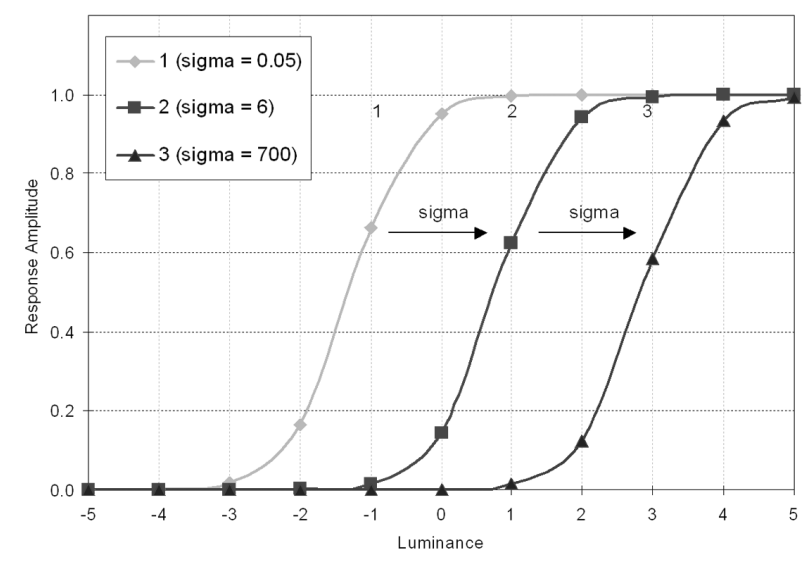

Figure 3: Multiplicative adaptation serves as a mechanism to maintain the sensitivity of the visual system.

Primarily, the adaptation occurs at the level of a localized post-receptoral site therefore the adapting value is dependent on the light that reaches individual regions of the retina. This means that a local model of adaptation is necessary if we want to accurately simulate the receptors' response to incoming light.

\subsection{A Static and Local Operator}

In our model we compute the separate local responses for rods and cones from a localized version of Equation 1:

$$
\begin{aligned}
R_{\text {cone }}(x, y) & =\frac{Y^{n}(x, y)}{Y^{n}(x, y)+\sigma_{\text {cone }}^{n}(x, y)} \\
R_{\text {rod }}(x, y) & =\frac{Y^{\prime n}(x, y)}{Y^{\prime n}(x, y)+\sigma_{\text {rod }}^{n}(x, y)}
\end{aligned}
$$

where $Y$ and $Y^{\prime}$ are the CIE photopic and scotopic luminance, and $\sigma$ is the half saturation parameter which is used to simulate the eye's multiplicative mechanism and is a function of the adaptation intensity.

To compute the adaptive mechanism $\sigma$, we use an empirical relationship originally proposed by Xie and Stockham [36]:

$$
\sigma=I_{A}^{\alpha} \times \beta
$$

where $I_{A}$ is the adaptation level and $\alpha$ and $\beta$ are constants and in our model differ for rods and cones.

Note that $\sigma$ is computed locally in our model since it is a function of a localized adaptation intensity $I_{A}(x, y)$ and is 
generated on a per-pixel basis from Equation 5:

$$
\left.\sigma_{(} x, y\right)=I_{A}^{\alpha}(x, y) \times \beta
$$

where $\alpha$ is 0.69 and $\beta$ is a value between 5.83 and $2.0 \mathrm{~cd} / \mathrm{m}^{2}$ depending on what receptor is being considered. In Equations 2 and 3 the photopic $(Y)$ and scotopic luminance $\left(Y^{\prime}\right)$ are computed by converting the input scene RGB (radiance) values (see [23]):

$$
\begin{gathered}
Y(x, y)=0.256 R+0.67 G+0.065 B \\
Y^{\prime}(x, y)=-0.702 R+1.039 G+0.433 B
\end{gathered}
$$

\subsection{Computing Local Adaptation}

Localized eye adaptation suggests using a local operator to compute the average local luminance $I_{A}(x, y)$ that appears in Equation 5. In our model, we use an edge preserving filter, referred to as the bilateral filter [9], to compute the average luminance on the neighborhood of each pixel and still avoid haloing artifacts. The bilateral filter is a non-linear filter where the output is a weighted average of the input. The weight of a pixel depends both on a standard Gaussian with spatial kernel $f$ and on an edge stopping function $g$ in the intensity domain, which decreases the weight of pixels with large intensity differences. The bilateral filter for a pixel $s$ is given by

$$
J(s)=\frac{1}{k(s)} \sum_{p \in n e i g h(s)} f(p-s) g\left(I_{p}-I_{s}\right) I_{p}
$$

where $k(s)$ is a normalization factor, given by

$$
k(s)=\sum_{p \in n e i g h(s)} f(p-s) g\left(I_{p}-I_{s}\right)
$$

A Gaussian is also used for $g$ in the intensity domain, as given by equation 10 . The value of the pixel $s$ is influenced mainly by pixels in the neighborhood that are close spatially and that have a similar intensity.

$$
g\left(I_{p}-I_{s}\right)=e^{-\frac{\left(I_{p}-I_{s}\right)^{2}}{2 * \sigma_{g}^{2}}}
$$

$\sigma_{g}$ is a scale parameter in the intensity domain that specifies what gradient intensity should stop diffusion. The bilateral filter is not prone to stairstepping artifacts; its output is smooth on a gradient input, therefore avoiding haloing artifacts. From empirical studies, we found out that $\sigma_{g}$ should change with $I_{s}$ and obtained good results with a wide range of input images for $\sigma_{g}=0.15 * I_{s}$. The bilateral filter is applied both to scotopic and photopic luminances, to determine each pixel adaptation luminances.

\subsection{Responsiveness of the Photoreceptors}

When the local adaptation level for given pixel is in photopic range, the model should generate a response signal for the cones only since the rods are completely saturated at any intensity above around $4 \mathrm{~cd} / \mathrm{m}^{2}$. In the scotopic condition the opposite obviously occurs because the cone system is not sensitive to scotopic levels. When the luminance of a region is within the mesopic range, both photoreceptors are sensitive and generate a response. To determine the saturation level of the photoreceptors at any given pixel, we compute as in [15] a mesopic factor mes $(x, y)$ as a transition on a log scale:

$$
\operatorname{mes}(x, y)=3\left(\frac{\log I_{A}(x, y)+2}{2.6}\right)^{2}-2\left(\frac{\log I_{A}(x, y)+2}{2.6}\right)^{3}
$$

where mes $(x, y)$ produces a mesopic map varying between [0..1] which is subsequently utilized to compute our final response for a given pixel. The correct computation of rod and/or cone response is fundamental because this will affect the perception of color in the tone mapped image: at scotopic light level we have no perception of colour and our ability to resolve fine detail is poor. Our local model allows us to compute for each pixel a response which is the result of stimulating cones, rods or a combination of the two, as shown in Figure 4.

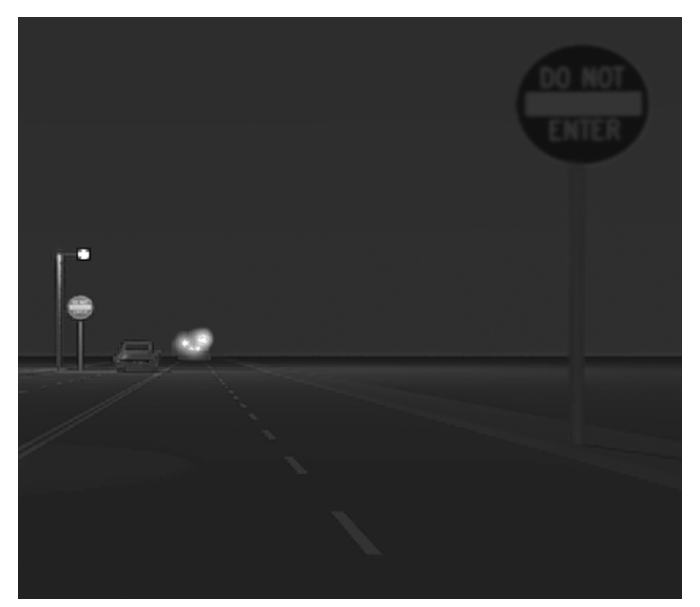

Figure 4: Night scene illustrating how our local model works. Under the bright light, the road sign appears red and we can even distinguish a blue car. However, we are not able to perceive the color of the closer sign on the right as it is too dark. Although the image is a simulation, it illustrates well the operator's behavior. Note that we have increased the brightness of the image for illustration purposes.

\subsection{Tone Mapping HDR Images}

Having computed a local response for both types of photoreceptors, we compute, similarly to [24], the inverse of the response function (Equation 1) in order to generate an output luminance channel for the rod and cones:

$$
\begin{aligned}
& Y_{\text {cone } O}(x, y)=\operatorname{mes}(x, y) \sigma_{\text {disp }}\left(\frac{R_{\text {cone }}(x, y)}{1-R_{\text {cone }}(x, y)}\right)^{(1 / n)} \\
& Y_{\text {rodo }}(x, y)=(1-\operatorname{mes}(x, y)) \sigma_{\text {disp }}\left(\frac{R_{\text {rod }}(x, y)}{1-R_{\text {rod }}(x, y)}\right)^{(1 / n)}
\end{aligned}
$$

To match the perception of visibility in the real scene with the visibility on the display, we first compute a global $\sigma$ value for the display which is a function of the viewer's adaptation level when observing the image on a low dynamic range device. A global value is adequate considering that the eye adaptation level is relatively constant when viewing an image on a display of limited luminance range. This $\sigma$ 

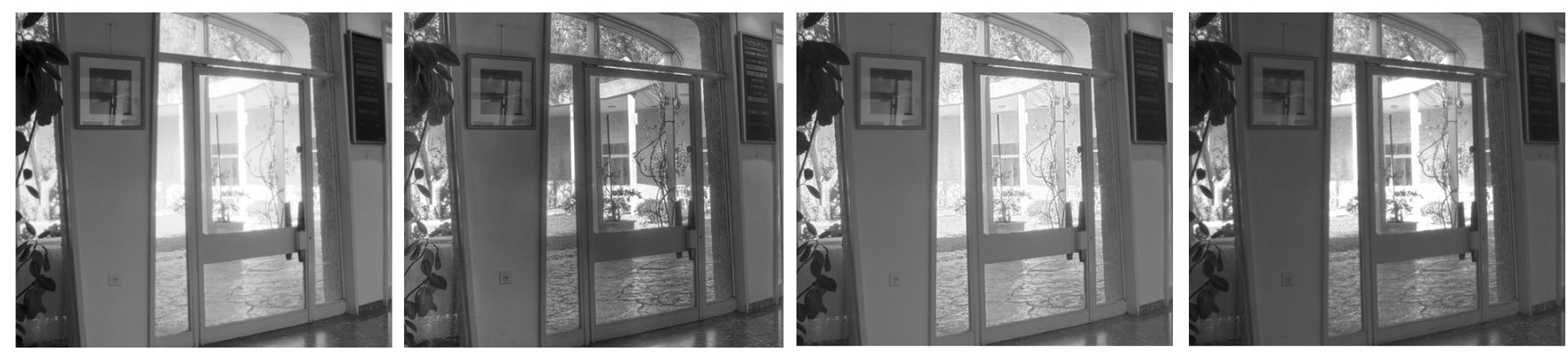

Figure 6: From left to right: Histogram [17] (global), Fattal-gradient [10](local), Photographic [25](local) and Our Model. Note how the visibility and contrast of the garden in Fattal et al.'s and our operator is higher compared to the other algorithms. Images courtesy of Dani Lischinski, Hebrew University, Israel.

is computed from Equation 4 where we use a value between 30 and $50 \mathrm{~cd} / \mathrm{m}^{2}$ as display adaptation level. After having computed the two luminance channels, $Y_{\text {coneO }}(x, y)$ is used to compute output RGB channels to which we finally sum $Y_{\text {rodo }}^{\prime}(x, y)$. In Figure 5 we compare our local model with Durand et al.'s Bilateral filter operator [9]. From this informal comparison it can be clearly seen that our operator does not suffer from halo or contrast reversal artifacts common in many local tone mapping operators. Figure 6 shows
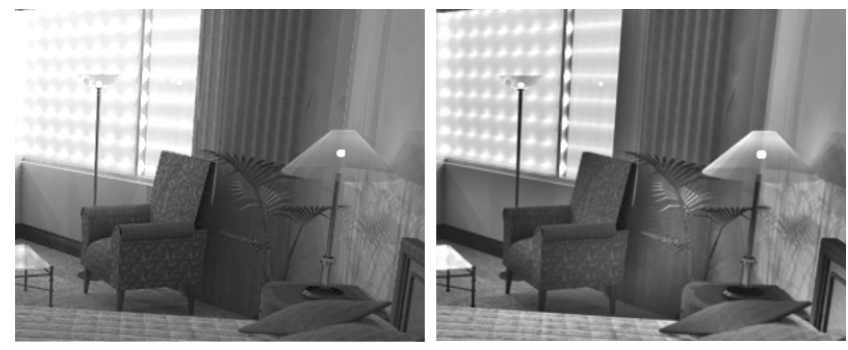

Figure 5: Informal comparison of our method (right) with Durand et al.'s Bilateral filter [9]. The new operator is capable of good contrast reduction and does not suffer from contrast reversal (see armchair and pillows). Image courtesy of Simon Crone, Perth, Australia.

comparisons of our model with some other tone mapping operators. Although we believe that making these comparisons may not be very meaningful, they illustrate the capabilities of our operator.

\section{EYE ADAPTATION}

Although the sensitivity of the eye changes as a function of the prevailing light, this process is not instantaneous. The mechanism by which the visual system changes its sensitivity, depending on the luminances in the visual field is known as adaptation. We refer to dark adaptation whenever there is a decrease in ambient illumination (e.g. when we enter a dark room from a brighter environment, or switch the light off) and light adaptation for the opposite effect. During dark adaptation the HVS recovers sensitivity which is experienced as temporary blindness, whereas when we undergo light adaptation we experience a loss in the system's sensitivity which allows us to see at the higher illumination levels. It would be a mistake to conclude that our vision is better at night of course; although our sensitivity decreases, the ability of the eye to discriminate different intensities increases in daylight. Our loss of sensitivity is the reason why we are not able to see the stars during daytime and our discrimination improvement can be noticed by the ability of detecting high spatial frequency detail on a background. This process of change in sensitivity takes time, however. The time-course of light and dark adaptation is well known and it is different for the cones and rods [14].

Figure 7 shows dark adaptation thresholds following preadaptation (the eye's adaptation level before the considered change) to five different background luminances $[12,13]$. Note that as the adapting intensity increases, the recovery of the eye's sensitivity is defined by two separate branches. The threshold drops rapidly in the first several minutes as the cone system is much faster in recovering its sensitivity. The cones reach their maximum sensitivity within 10-12 minutes although this is still relatively high, as can be seen in Figure 7. After approximately 10 minutes the rods become more sensitive to the adapting low light and a transition from detection of the cones to the rods occurs which is experienced by a loss of color. The rod system takes about 40 minutes to fully recover although this is related to various preadapting factors. From Figure 7, it may seem that dark adaptation is solely a function of the intensity of the preadapting light however this is not true. Adaptation is also affected by the duration of the preadapting light. As the preadapting time increases, the longer the recovery of sensitivity. This is very important when trying to develop a model that simulates eye adaptation as we need to be aware not only of how bright (or dark in case of light adaptation) an environment was before the change in illumination, but also for how long we were preadapted.

The time-course of light adaptation of the cone system is shown in Figure 8. The main difference between dark and light adaptation is the time it takes for the system to reach equilibrium. Note that the thresholds are highest immediately after the onset of a bright adapting field and decrease very rapidly within the first few seconds. The complete equilibrium is reached after around three minutes. Figure 8 shows the time-course of adaptation for two different adapting intensities; although there is a difference in absolute threshold values, the drop is in both cases of around 0.5 log and the time-course is very similar. 


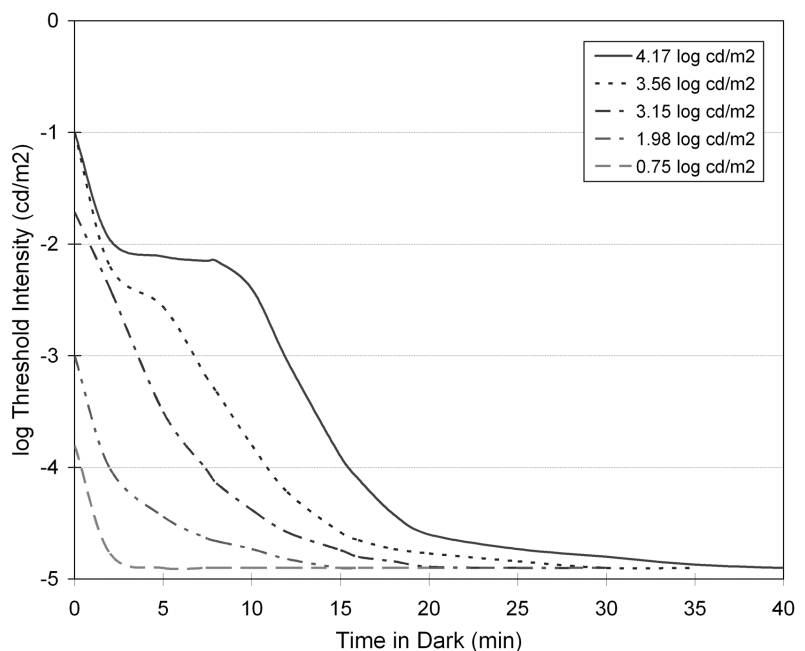

Figure 7: The time-course of dark adaptation as a function of preadapting luminance. With time the threshold decreases and we experience an improvement in visibility. After Haig and Hecht $[12,13]$

\subsection{A Time Dependent Model}

Our operator is not only capable of significant contrast compression allowing us to tone map HDR images, but it also simulates dark and light adaptation if a frame sequence of a scene with a change in lighting is given. The model is derived from widely accepted psychophysical data. Since the $\sigma$ parameter in Equations 2 and 3 is used as an adaptation mechanism, we can employ once more the electrophysiological model to simulate the time-course of adaptation by varying this variable according to some specific criteria. However, in doing so there are three main issues that need to be considered: firstly how do we interpret and model the psychophysically-based threshold data related to adaptation (see Figures 7 and 8 for example)? Secondly, how do we vary $\sigma$ to simulate such adaptation? And finally, how do we generate the appropriate shape and duration of the adaptation curve? We will deal with these one at a time.

\subsection{A Model of the Timecourse of Adaptation}

Figures 7 and 8 present detection thresholds during adaptation. Our response model computes the response amplitude for a given pixel which is then utilized to compute the output luminance. The time-course of adaptation is approximately a reciprocal exponential function (Figures 7 and 8) signifying that our visibility of an adapting image (frame sequence) on the display should follow a similar shape. However, when attempting to model dark adaptation, for instance, we are trying to simulate the perception of the scene from a viewer's perspective. When an observer is dark adapting, the visibility on the scene will be very low at first and the viewer is temporarily blinded (except for very bright objects of course). From the time-course of adaptation, we can see that with the passing of time, the detection threshold becomes lower as the result of the photoreceptors' sensitivity being recovered. We visually experience this as an improvement of visibility and those objects which previously would appear black, become more detectable (see Figure 9). The

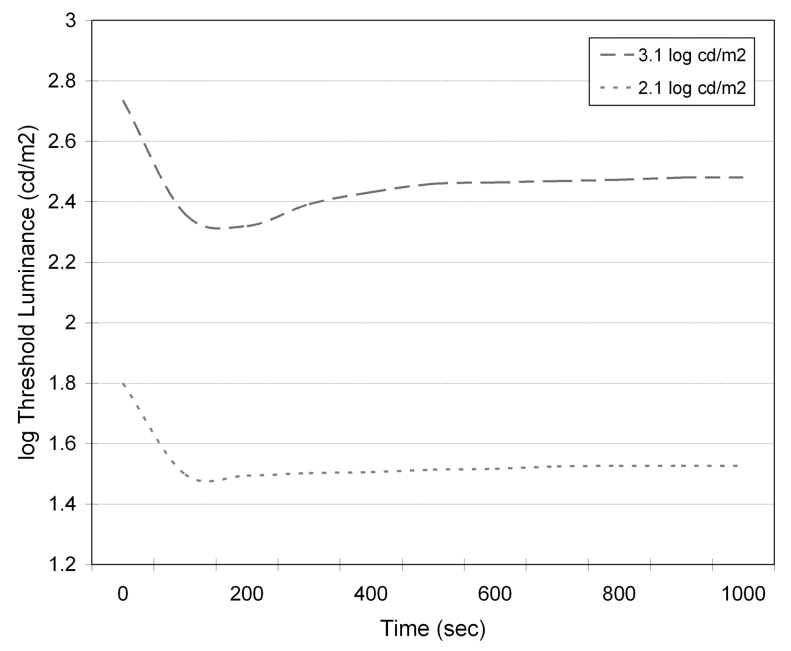

Figure 8: The time-course of light adaptation as a function of preadapting luminance. After Baker [2]

recovery of visibility closely follows a logarithmic curve.

Having established that the time-course of adaptation can be simulated by producing incremental frames in which each pixel's intensity grows logarithmically, the issue then becomes to determine how to vary $\sigma$ in order to produce such output. Our static tone mapping operator assumes that the viewer is fully adapted to the scene, therefore, given an initial and final adaptation states, the operator would generate a different $\sigma$ for each image ( $\sigma$ is a function of the adaptation level). Note that the model does not necessarily require abrupt changes in luminance from initial and final state but also functions with smaller and continuous changes however the following discussions mainly refers to the circumstances where we consciously experience adaptation. We call $\sigma_{i n}(x, y)$ and $\sigma_{f i n}(x, y)$ the $\sigma$ value at the preadaptation level (initial value) and fully-adapted level(final) respectively. We derived an empirical formulae which interpolates between $\sigma_{i n}(x, y)$ and $\sigma_{f i n}(x, y)$ as a function of time. At each frame a new $\sigma_{t}(x, y)$ is computed which we then use to compute the retinal response at that frame. The formulae for dark adaptation for cones and rods are presented respectively below:

$\sigma_{t}(x, y)=$

$\sigma_{f i n}(x, y)+\frac{\text { OONE }_{\text {dark }- \text { posdark }_{\text {fin }}}}{\text { posdark }_{i n}-\text { posdark }_{\text {fin }}} \times\left(\sigma_{i n}(x, y)-\sigma_{f i n}(x, y)\right)$

where:

$$
C O N E_{\text {dark }}=\sigma_{i n}(x, y) \exp ^{K / t}
$$

$\sigma_{t}(x, y)$ is the value of $\sigma$ after time $t$ into the adaptation process. The variables posdark pin $_{\text {and }}$ asdark fin are simply the values that $\sigma$ would assume at the initial and final position of the time-course curve. These constants are just used as normalizing factors (see Table 1). The dark adaptation curve for the rod system is very similar to Equation 14 except that we substitute $C O N E_{\text {dark }}$ with $R O D_{\text {dark }}$ :

$$
R O D_{\text {dark }}=\sigma_{\text {in }}(x, y) \exp ^{K /\left(t-\tau_{\text {cone }}\right)}
$$



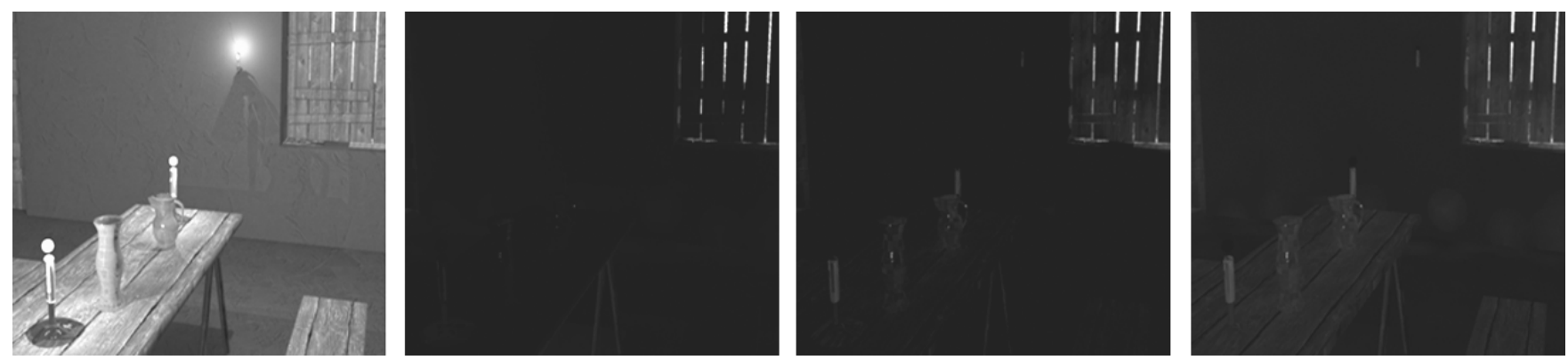

Figure 9: Simulating dark adaptation. After being adapted to a high ambient level (frame 1), the lights are switched off. At first (frame 2) we experience a loss of visibility as our visual system has yet to recover and become sensitive to such low light levels. After some time (this is mainly a function of pre-adapting luminance and pre-adapting time), sensitivity increases allowing us to recover some visibility. Note that we have increased the brightness of the image for illustration purposes.

Simulating the time-course of light adaptation is similar. We still use the same structure as in Equation 14 however, in this circumstance we substitute $C O N E_{\text {dark }}$ with $C O N E_{\text {light }}, R O D_{\text {dark }}$ with $R O D_{\text {light }}$ and the normalizing variables:

$\sigma_{t}(x, y)=$

$\sigma_{f i n}(x, y)+\frac{\text { CONE }_{\text {light }}-\text { poslight }_{f i n}}{\text { poslight }_{\text {in }}-\text { poslight }_{\text {fin }}} \times\left(\sigma_{\text {in }}(x, y)-\sigma_{f i n}(x, y)\right)$

where:

$$
\begin{gathered}
C O N E_{\text {light }}=\sigma_{\text {in }}(x, y) K \times \log (t) \\
R O D_{\text {light }}=\sigma_{\text {in }}(x, y) K \times \log (t)
\end{gathered}
$$

$C O N E_{\text {light }}$ and $R O D_{\text {light }}$ are computed using the $\log (t)$ instead of the exponential. This is because, visually, light adaptation is the opposite of dark adaptation. Note that in the Equation 14 and subsequent ones we omitted, for practical purposes, the subscript cone or rod required with $\sigma(x, y)$. The appropriate should be used according to the numerator of Equations 14 and 15.

From the above it may appear that the model requires an initial and final state and then computes all the intermediary adaptation stages. More specifically, the operator requires an initial and final state to predict the time course of adaptation if no other change in lighting would occur in the scene. However, on a frame basis, the model determines if a new change in illumination did occur and adjusts the adaptation process (by setting new initial and final states) accordingly. Therefore we are capable of simulating not only abrupt and single changes in luminance but continuos variation in a sequence.

Adaptation is not only a function of intensity, the knowledge of the pre-adaptation duration is also fundamental. In the previous equations we use the variable $K$ to determine the shape of the curve, once we have established whether we are dark or light adapting. The value of $K$ is derived from an experiment by Mote and Riopelle [19]. They tested the timecourse of dark adaptation as a function of both preadapting luminance and time. They concluded that over a wide range of duration and intensities an increase in one is equivalent to increasing the other. The important practical question they were trying to answer was what combination of the product

$$
E=\text { Intensity } \times \text { PreAdaptationTime }
$$

produced a unique dark adaptation curve. $E$ is energy. From their data they concluded that below $10567 \mathrm{~cd} / \mathrm{m}^{2} \mathrm{sec}$ (converted from their original units of $3320 \mathrm{mLsec}$ ) this product can be used to determine the correct adaptation curve.

This result is very interesting because from the computation of the Energy product (our model keeps track of preadaptation time and intensity) we can determine with sufficient level of accuracy the time-course of adaptation for a particular scene. At this stage, it is important to mention that there are many other factors that affect the timecourse of adaptation such as the wavelength distribution of the light, pigment regeneration and size and position of the test targets which we will consider in the future. From the parameter $E$ we can therefore estimate how steep the adaptation curve will be; this can be used to compute the value $K$, derived by linear interpolation:

$$
K=-0.0006 E+12.0
$$

Equation 17 derives from linearly interpolating between the maximum and minimum energy products. The larger $\mathrm{E}$ the smaller the value of $\mathrm{K}$, therefore adjusting the convergence curve so that adaptation time increases up to a maximum of 40 minutes.

\section{RESULTS}

In Figure 12 we present some of the results of our model. Although presenting images in this way is not possibly the

\begin{tabular}{|c|c|c|c|}
\hline Dark Ad & posdark $_{\text {in }}$ & posdark $_{\text {fin }}$ & t const $(\mathrm{sec})$ \\
\hline $\begin{array}{l}\text { cones } \\
\text { rods }\end{array}$ & $\begin{array}{l}\sigma_{i n} \exp ^{K} \\
\sigma_{i n} \exp ^{K}\end{array}$ & $\begin{array}{c}\sigma_{\text {in }} \exp ^{K / \tau_{\text {cone }}} \\
\sigma_{\text {in }} \exp ^{K / \tau_{\text {rod }}}\end{array}$ & $\begin{array}{l}\tau_{\text {cone }}=600 \\
\tau_{\text {rod }}=2400\end{array}$ \\
\hline Light Ad & poslight $_{\text {in }}$ & $\overline{\text { poslight }}_{\text {fin }}$ & $\mathrm{t}$ const \\
\hline $\begin{array}{l}\text { cones } \\
\text { rods }\end{array}$ & $\begin{array}{l}0 \\
0\end{array}$ & $\begin{array}{c}\sigma_{\text {in }} K \times \log \left(\rho_{\text {cone }}\right) \\
\sigma_{\text {in }} K \times \log \left(\rho_{\text {rod }}\right)\end{array}$ & $\begin{array}{c}\rho_{\text {cone }}=20 \\
\rho_{\text {rod }}=20\end{array}$ \\
\hline
\end{tabular}
most accurate way to asses the operator, we still believe that

Table 1: Table containing all the normalizing factors and time constants 
it would be interesting to show the contrast compression of some popular HDR images. The two sequences show dark and light adaptation respectively.

\subsection{Validating the Model}

Tone mapping operators should produce images which closely resemble the viewer's perception of the scene as if he was standing in the real environment. Although we show some comparisons with other operators we believe that this is less meaningful than the ideal of determining the accuracy of our model by making comparisons with the real scene. To make comparison with the actual environment that the scene is trying to represent can be very challenging firstly because we do not always have access to the real scene and secondly because running such experiments is not an easy task. However, we have access to a HDR display which is capable of displaying linearly a large range of luminance levels. This device allowed us to directly test our operator displayed on a standard CRT monitor against the HDR device [28]. We ran a pilot study (similar to [18]) where we tested contrast charts displayed on the high dynamic range monitor against the same images tone mapped and displayed on a typical monitor. During the trial, participants had to observe in a darkened room a series of test-stimuli displayed with our method and make comparisons with the reference stimuli linearly displayed on the HDR monitor. We tested how perceptually similar the reproduction of fine detail and contrast were. The results show a strong correlation between the images created using our model displayed on a standard monitor and those linearly mapped on the HDR display. A more formal validation will be conducted in the future.

In the validation mentioned above, only static images were tested. These images, like for most tone mapping operators, assume that the viewer in the scene is fully adapted. Our model however is also capable of simulating eye adaptation. We tested the accuracy of our dark adaptation model by reproducing a tvi experiment. We created a set of 10 scenes of a constant low-luminance background level, each containing a test target in the center of different luminance levels (Figure 10). We ran our model with each scene, firstly providing as input a white image (to ensure light adaptation) and subsequently turning the white light off and displaying on of the 10 scenes for 40 minutes. Our model then based on the initial light adapted state and final state, generated a number of frames to simulate dark adaptation over the 40 minutes period. As it happens in reality, the bright targets were visible immediately whereas the dimmer targets at first appeared black and with time became more visible as the threshold lowered. For each scene, we then asked a small group of participants, to observe the frames generated and say at which frame (representing time) they started seeing the target. We plotted how long it takes, during the dark adaptation process, for the target input luminance to become noticeable from the background. According to Figures 7 and the results from Mote and Riopelle [19], thresholds fall with time as a function of energy $E$. To test our model we plotted the detection of the target as a function of time the results of which are presented in Figure 11. From these results it can be seen how our data matches closely with the psychophysical experimental data shown in Figure 7.

\section{CONCLUSIONS AND FUTURE WORK}
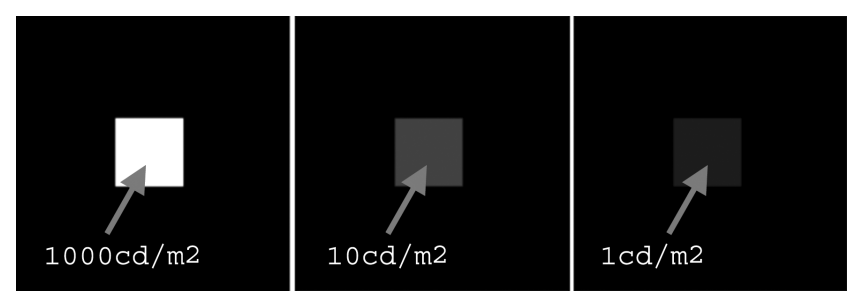

Figure 10: Three of the ten Targets used for the validation of dark adaptation. Although, when fully adapted we are able to see immediately the targets, during adaptation this takes time and is a function of preadaptation time and intensity as well as a function of the actual target's luminance.

Dark Adaptation

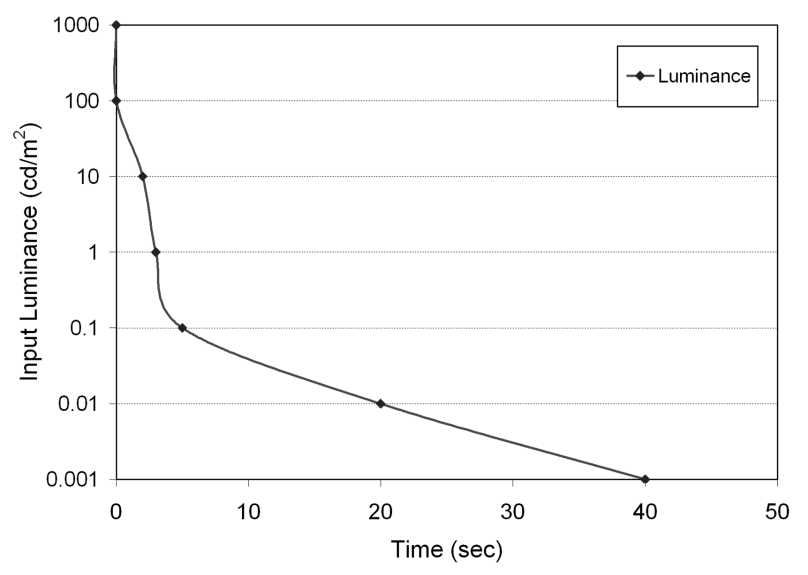

Figure 11: Experimental data produced by our operator: Minimum Input Luminance noticeable from the background at each instant during dark adaptation.

In the natural world, the eye is confronted with a huge range of luminances. Eye adaptation is the mechanism that allows our eyes, by changing their sensitivity, to be responsive at varying illumination levels. Thanks to this highly localized mechanism, our visual system is able to see luminances at extreme ends. We have presented a new local model of adaptation and tone mapping to compress HDR static images as well as frame sequences. The operator derived from this model is completely automatic, in the sense that no special parameterization was required for the large set of images tested. The model is new as it allows us to adapt each pixel, both in space and time, differently based on a neighborhood of values: it can cope well with complex HDR images and is able to handle photopic, mesopic and scotopic conditions simultaneously for different regions of the same image. The model simulates the time course of adaptation as a function of pre-adaptation time and intensity, adjusting the converge curve in accordance to these parameters. The experiments made to compare the operator output with images displayed on a HDR monitor show a strong correlation between both.

Our model could be improved by including spectral rendering, which would allow a more accurate computation of the 
rods and cones sensitivity, especially in scotopic levels where there is shift in the eye sensitivity to shorter wavelengths. Individual responses of the long, medium and short conetypes could also be computed to simulate more accurately local adaptation. The introduction of secondary effects such as glare and veiling [29, 17] would enhance the realism especially for animations. Finally, as with all tone mapping operators, we believe that a more formal psychophysical validation needs to take place to asses the quality of our model against other similar operators and the real scenes they are attempting to portray.

\section{REFERENCES}

[1] M. Ashikhmin. A tone mapping algorithm for high contrast images. In In 13th Eurographics Workshop on Rendering. Eurographics, June 2002. 10, 15, 2002.

[2] H. Baker. The course of foveal light adaptation meausred by threshold intensity increment. Journal of Optometry, 39:172-179, 1949.

[3] K. Chiu, M. Herf, P. Shirley, S. Swamy, C. Wang, and K. Zimmerman. Spatially Nonuniform Scaling Functions for High Contrast Images. In Proceedings of Graphics Interface '93, pages 245-253, San Francisco, CA, 1993. Morgan Kaufmann.

[4] P. Choudhury and J. Tumblin. The trilateral filter for high contrast images and meshes. In Eurographics Workshop on Rendering, pages 1-11, 2003.

[5] P. E. Debevec and J. Malik. Recovering high dynamic range radiance maps from photographs. Computer Graphics, 31(Annual Conference Series):369-378, 1997.

[6] J. Dicarlo and B. Wandell. Rendering high dynamic range images. In SPIE: Image Sensors, pages 392-401, 2000.

[7] F. Drago, K. Myszkowski, T. Annen, and N. Chiba. Adaptive logarithmic mapping for displaying high contrast scenes. In Eurographics 2003, 2003.

[8] F. Durand and J. Dorsey. Interactive tone mapping. In B. Peroche and H. Rushmeier, editors, Rendering Techniques 2000 (Proceedings of the Eleventh Eurographics Workshop on Rendering), pages 219-230, New York, NY, 2000. Springer Wien.

[9] F. Durand and J. Dorsey. Fast bilateral filtering for the display of high dynamic range images. In $A C M$ Transactions on Graphics (Proceedings of SIGGRAPH'2002, pages 257-266, 2002.

[10] R. Fattal, D. Lischinski, and M. Werman. Gradient domain high dynamic range compression, 2002.

[11] J. A. Ferwerda, S. N. Pattanaik, P. Shirley, and D. P. Greenberg. A model of visual adaptation for realistic image synthesis. Computer Graphics, 30(Annual Conference Series):249-258, 1996.

[12] C. Haig. The course of rad dark adaptation as influenced by the intensity and duration of preadaptation to light. Journal of general Physiology, 24:735-751, 1941.

[13] S. Hecht, C. Haig, and A. M. Chase. The influence of light-adaptation on subsequent dark-adapatiotion of the eye. Journal of general Physiology, 20:831-8501, 1937.

[14] D. Hood and M. Finkelstein. Sensitivity to light. Handbook of Perception and Human Performance, 1(5):1-66, 1986.

[15] H. W. Jensen, S. Premoze, P. Shirley, W. B. Thompson, J. A. Ferwerda, and M. M. Stark. Night rendering technical report, 2000.

[16] D. J. Jobson, Z. ur Rahman, and G. A. Woodell. A multiscale retinex for bridging the gap between color images and the human observation of scenes. IEEE Transactions on Image Processing, 6(7):965-976, July 1997.
[17] G. W. Larson, H. Rushmeier, and C. Piatko. A visibility matching tone reproduction operator for high dynamic range scenes. IEEE Transactions on Visualization and Computer Graphics, 3(4):291-306, /1997.

[18] P. Ledda, G. Ward, and A. Chalmers. A wide field, high dynamic range, stereographic viewer. GRAPHITE 2003, pages 237-244, 2003.

[19] F. A. Mote and A. J. Riopelle. The effect of varying the intensity and the duration of preexposure upon foveal dark adaptation in the human eye. J. comp. physiol. Psychol., 46(1):49-55, 1953.

[20] K. I. Naka and W. A. H. Rushton. S-potential from colour units in the retina of fish (cyprinidae). Journal of Physiology, 185:536-555, 1966.

[21] B. Oh, M. Chen, M. Dorsey, and F. Durand. Image-based modeling and photo editing. In $S I G G R A P H$, pages 433-442, 2001.

[22] S. Pattanaik and H. Yee. Adaptive gain control for high dynamic range image display. In Proceedings of the 18th spring conference on Computer graphics, pages 83-87. ACM Press, 2002.

[23] S. N. Pattanaik, J. A. Ferwerda, M. D. Fairchild, and D. P. Greenberg. A multiscale model of adaptation and spatial vision for realistic image display. Computer Graphics, 32(Annual Conference Series):287-298, 1998.

[24] S. N. Pattanaik, J. Tumblin, H. Yee, and D. P. Greenberg. Time-dependent visual adaptation for fast realistic image display. In SIGGRAPH, pages 47-54, 2000.

[25] E. Reinhard, M. Stark, P. Shirely, and J. Ferweda. Photographic tone reproduction for digital images. In SIGGRAPH, 2002.

[26] A. Scheel, M. Stamminger, and H.-P. Seidel. Tone reproduction for interactive walkthroughs. Computer Graphics Forum, 19(3), 2000.

[27] C. Schlick. Quantization techniques for the visualization of high dynamic range pictures. In Eurographics Workshop on Rendering, pages 7-20, 1994.

[28] H. Seetzen, L. Whitehead, and G. Ward. A high dynamic range display system using low and high resolution modulators. In Proc. of the 2003 Society for Information Display Symposium, 2003.

[29] G. Spencer, P. Shirley, K. Zimmerman, and D. P. Greenberg. Physically-based glare effects for digital images. Computer Graphics, 29(Annual Conference Series):325-334, 1995.

[30] J. Tumblin, J. Hodgins, and B. Guenter. Two methods for display of high contrast images. ACM Transactions on Graphics, 18(3):56-94, 1999.

[31] J. Tumblin and H. Rushmeier. Tone reproduction for computer generated images. IEEE Computer Graphics and Applications, 13(6):42-48, 1993.

[32] J. Tumblin and G. Turk. LCIS: A boundary hierarchy for detail-preserving contrast reduction. In A. Rockwood, editor, Siggraph 1999, Computer Graphics Proceedings, pages 83-90, Los Angeles, 1999. Addison Wesley Longman.

[33] S. Upstill. The realistic presentation of synthetic images: Image processing in computer graphics, 1985.

[34] G. Ward. A contrast-based scalefactor for luminance display. Graphics Gems IV, pages 415-421, 1994.

[35] G. Ward and R. A. Shakespeare. Rendering with radiance. 1998.

[36] Z. Xie and T. G. Stockham. Towards the unification of three visual laws and two visual models in brightness perception. IEEE Transactions on Systems, Man, and Cybernetics, 19:379-387, 1989. 

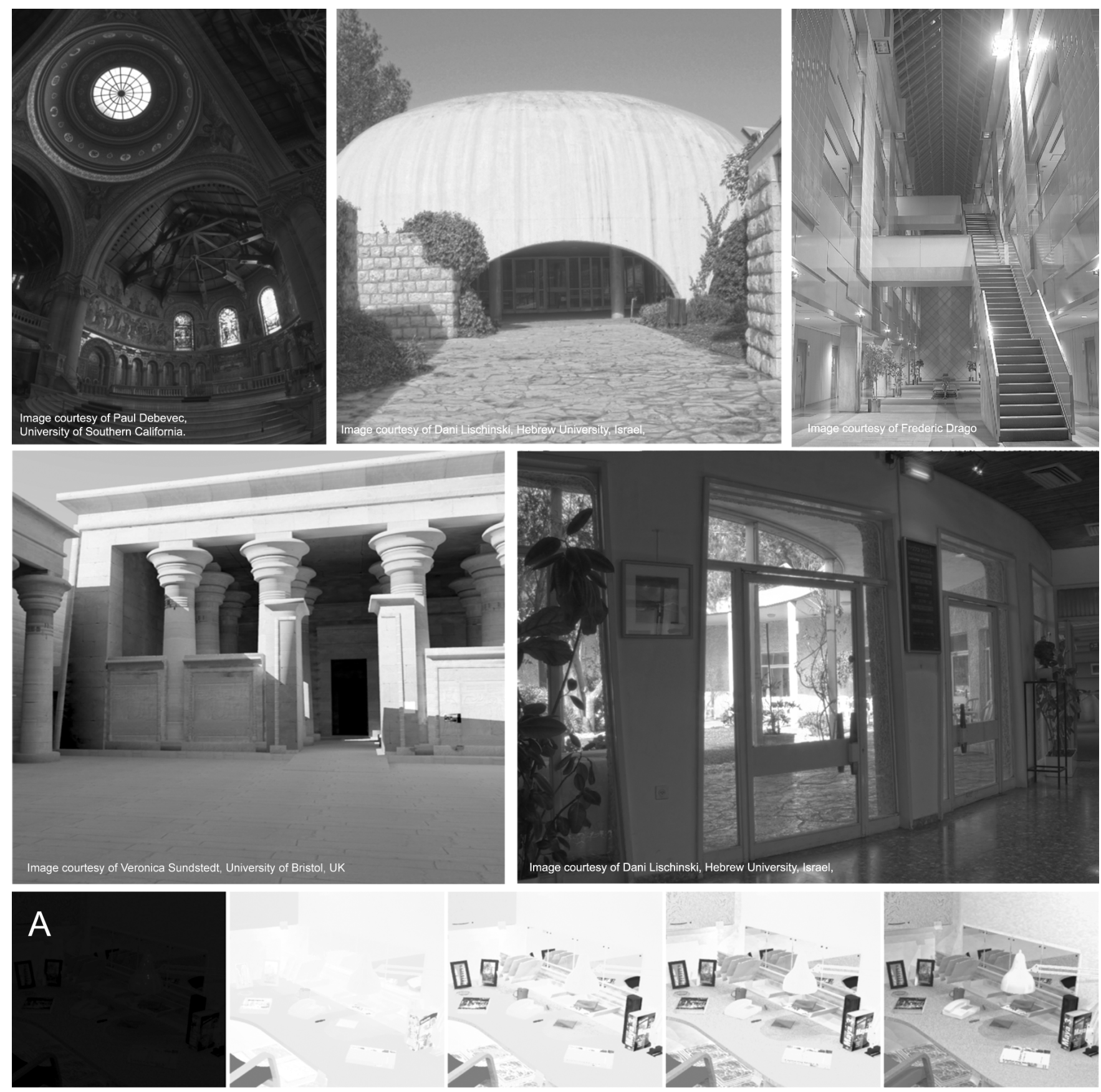

Image courtesy of Dani Lischinski, Hebrew University, Israel,
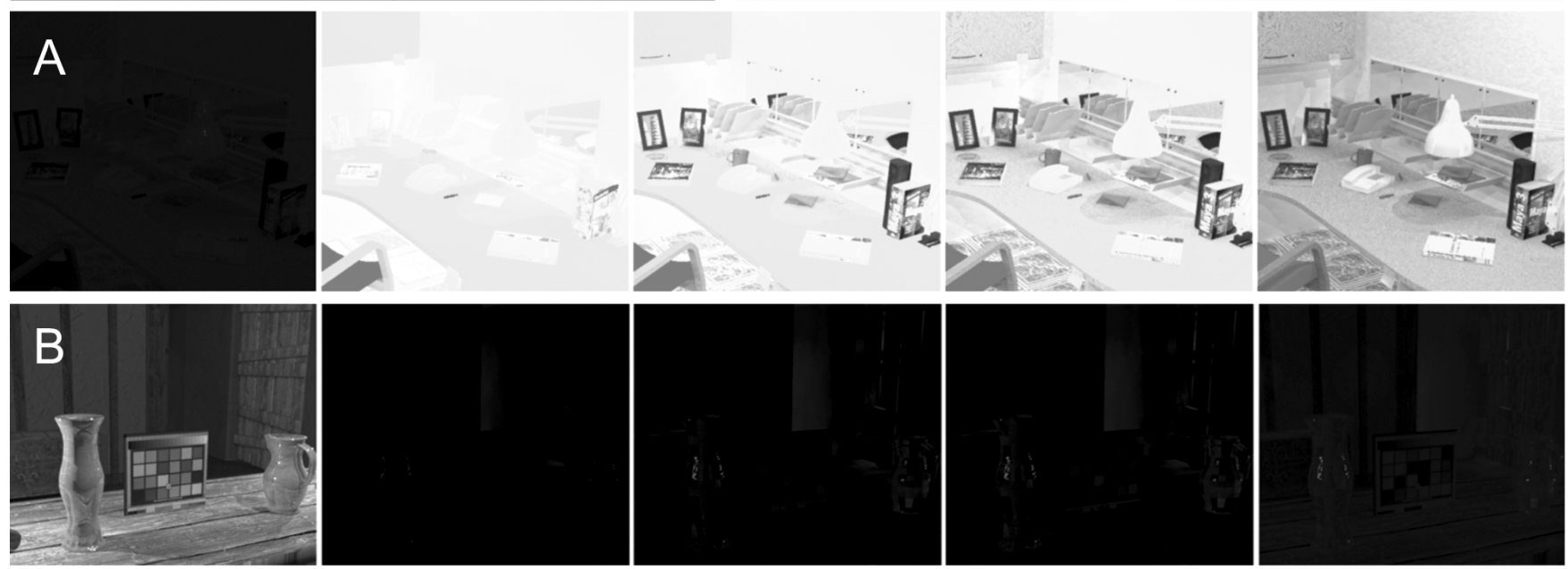

Figure 12: The top five images are tone-mapped scenes using the model in this paper. Sequence A shows an example of light adaptation after being preadapted to a dark environment. The sequence represent light adaptation after 1,3,6 and 12 seconds respectively. In Sequence B we show some frames from a dark adaptation example. After being fully adapted to a high illumination, the lights are switched off. The following four frames show the perception of the scene after 1,2,4,16 minutes. From the computation of Energy E, our model determines the speed of adaptation. In this case of dark adaptation, $\mathrm{E}$ is not very high. As a consequence, our visual system adapts fairly quickly. Also, note the difference in adaptation times between the two sequences. 